\title{
VÍTIMAS DE ACIDENTES DE TRÂNSITO ATENDIDAS POR SERVIÇO PRÉ-HOSPITALAR MÓVEL DE URGÊNCIA
}

\section{VICTIMS OF TRAFFIC ACCIDENTS ATTENDED BY PREHOSPITAL MOBILE EMERGENCY CARE}

\section{VÍCTIMAS DE ACCIDENTES DE TRÁNSITO ATENDIDAS POR SERVICIO PRE-HOSPITALAR MÓVIL DE URGENCIA}

Rodrigo Assis Neves Dantas ${ }^{1}$, Lívia Maria Nunes Henriques ${ }^{2}$, Daniele Vieira Dantas ${ }^{3}$, Sara Porfírio de Oliveira ${ }^{2}$, Sabrina Daiane Gurgel Sarmento $^{4}$

\section{RESUMO}

Objetivo: descrever o perfil epidemiológico das vítimas de acidentes de trânsito socorridas por um Serviço de Atendimento Móvel de Urgência. Método: estudo exploratório, descritivo, de abordagem quantitativa dos dados. A coleta foi realizada de janeiro a junho de 2016 em hospital referência em atendimento de urgência e emergência. Resultados: foram analisadas 125 ocorrências de Acidentes de Trânsito, sendo 101 (80,8\%) do sexo masculino, com faixa etária entre 18 a 73 anos. Desses, 49,6\% estudaram até o Ensino Médio. A Unidade de Suporte Básico à Vida foi utilizada em $87,2 \%$ dos casos relatados e $42,4 \%$ das vítimas foram socorridas em tempo inferior a 20 minutos, 84\% sofreram colisão e 50 (40\%) dos acidentes ocorreram pela manhã. Conclusão: a caracterização deste perfil gera subsídios para elaboração de estratégias visando melhorar a qualidade da assistência prestada.

Descritores: Serviços médicos de emergência; Assistência pré-Hospitalar; Acidentes de trânsito; Perfil epidemiológico; Enfermagem em emergência.

\begin{abstract}
Objective: to describe the epidemiological profile of victims of traffic acidents rescued by a Mobile Emergency Care Service. Method: exploratory, descriptive study, with quantitative data approach. Collection occurred from January to June 2016 at a hospital reference in urgency and emergency care. Results: 125 reports of Traffic Accidents were analyzed, of which 101 (80.8\%) were males, aged between 18 and 73 years. Of them, 49.6\% studied until High School. The Basic Life Support Unit was used in $87.2 \%$ of the reported cases and $42.4 \%$ of the victims were rescued in less than 20 minutes, $84 \%$ suffered collision and 50 (40\%) accidents occurred in the morning. Conclusion: the characterization of this profile subsidizes the elaboration of strategies aimed at improving the quality of the provided care.
\end{abstract}

Descriptors: Emergency medical services; Pre-hospital care; Traffic accidents; Epidemiological profile; Emergency Nursing.

\section{RESUMEN}

Objetivo: describir el perfil epidemiológico de las víctimas de accidentes de tránsito socorridas por un Servicio de Atención Móvil de Urgencia. Método: estudio exploratorio, descriptivo, de abordaje cuantitativo de los datos. La recolección fue realizada desde enero a junio de 2016 en un hospital de referencia en atención de urgencia y emergencia. Resultados: se analizaron 125 casos de accidentes de tráfico, siendo 101 (80,8\%) del sexo masculino, con edad entre 18 y 73 años. De ellos, 49,6\% estudiaron hasta la Enseñanza Media. La Unidad de Soporte Básico a la Vida fue utilizada en el 87,2\% de los casos reportados y el $42,4 \%$ de las víctimas fueron socorridas en tiempo inferior a 20 minutos, $84 \%$ sufrieron colisión y 50 (40\%) accidentes ocurrieron por la mañana. Conclusión: la caracterización de este perfil genera subsidios para la elaboración de estrategias para mejorar la calidad de la asistencia prestada.

Descriptores: Servicios médicos de urgencia; Atención prehospitalaria; Accidentes de tránsito; Perfil epidemiológico; Enfermería de urgência.

\footnotetext{
${ }^{1}$ Graduado em Enfermagem. Pós-Doutorando em Enfermagem pela Universidade Federal de Sergipe. Docente na Universidade Federal do Rio Grande do Norte. ${ }^{2}$ Graduanda em Enfermagem pela Universidade Federal do Rio Grande do Norte. ${ }^{3}$ Graduada em Enfermagem. Pós-Doutora em Enfermagem pela Universidade Federal de Sergipe. Docente na Universidade Federal do Rio Grande do Norte. ${ }^{4}$ Graduada em Enfermagem pela Universidade Federal do Rio Grande do Norte.
} 


\section{INTRODUÇÃO}

Acidentes são considerados um problema de saúde púbica de grande magnitude e transcendência, devido ao seu alto índice de morbimortalidade $^{(1)}$. Em 2015, segundo a OMS, aproximadamente 1,3 milhão de pessoas morreram no mundo, vítimas deste tipo de acidente e, até 50 milhões sofreram algum tipo de lesão ${ }^{(1)}$. No Brasil, mais de 35 mil pessoas morrem por ano, 120 mil necessitam ser hospitalizadas no setor público devido a Acidentes de Trânsito (AT) ${ }^{(2)}$.

O Instituto de Pesquisa Econômica Aplicada (IPEA) e a Associação Nacional de Transportes Públicos (ANTP) apontam vários impactos negativos para a saúde da população decorrentes de acidentes de trânsito. São eles: perda de anos de vida livres de incapacidade, redução da expectativa de vida dos adolescentes e jovens, além dos altos custos sociais, judiciais e econômicos gerados ao sistema de saúde e previdenciário $^{(3)}$.

Diante dessa grande demanda, em 2003, foi criado o Serviço de Atendimento Móvel de Urgência (SAMU), instituído pela Política Nacional de Atenção às Urgências, através da Portaria no1863/GM $\mathrm{GM}^{(4)}$. O SAMU é um serviço préhospitalar, cujo objetivo é um atendimento precoce e eficaz no transporte para um serviço de urgência e emergência e tem a capacidade de atender $75 \%$ da população brasileira, localizada em quase 3 mil municípios ${ }^{(5)}$.

Os acidentes de trânsito ocorrem em grande expansão devido ao aumento desordenado de veículos nas vias e projetos de urbanismo desatualizados. No século $X X$, com o desenvolvimento industrial, houve aumento importante na produção e utilização de veículos automotivos. Somado a isso, projetos de urbanismo desatualizados, corroborando assim o aumento de acidentes onde têm seu primeiro atendimento realizado pelo SAMU ${ }^{(6)}$.

Este estudo justifica-se pela necessidade de aprofundar o conhecimento acerca dos principais acidentes de trânsito e suas vítimas, na busca de proporcionar maior reflexão e discussão entre os profissionais de saúde e usuários sobre a temática, fortalecendo as políticas públicas de saúde preconizadas pelo SUS. Diante disso, o presente estudo tem como objetivo descrever o perfil epidemiológico das vítimas de acidentes de trânsito socorridas por um Serviço de Atendimento Móvel de Urgência.

\section{MÉTODO}

Trata-se de um estudo exploratório, descritivo e de abordagem quantitativa dos dados, realizado no Pronto Socorro Clóvis Sarinho (PSCS), referência no atendimento de urgência e emergência no estado do Rio Grande do Norte (RN), que faz parte do Complexo Hospitalar Monsenhor Walfredo Gurgel (HMWG), localizado na cidade Natal/RN.

O presente estudo possui uma amostra de conveniência de 125 vítimas de acidentes de trânsito, atendidas pelo Serviço de Atendimento Móvel de Urgência 192 do estado do Rio Grande do Norte (SAMU 192 RN), após serem estabilizadas e transportadas para o PSCS.

Para serem incluídos nesta pesquisa, eram necessários os seguintes critérios: apresentar idade igual ou superior a 18 anos, estar consciente e orientado ou acompanhado por responsável legal em caso de inconsciência. Foram excluídas as vítimas que sofreram traumas não relacionados a acidentes de trânsito.

A coleta de dados ocorreu no período de janeiro a junho do ano de 2016, por meio da aplicação de um instrumento estruturado composto pelas seguintes questões: características sociodemográficas (sexo, faixa etária e grau de escolaridade), tempo resposta, qual o tipo de veículo utilizado para o transporte da vítima: Unidade de Suporte Básico (USB) ou Unidade de Suporte Avançado (USA), qual o tipo de acidente (colisão, queda, capotamento ou atropelamento) e ainda qual área do corpo foi lesionada além do dia da semana e turno em que o AT ocorreu.

Após serem atendidos pelo SAMU $192 \mathrm{RN}$ e estabilizados no PSCS, a vítima e/ou responsável legal eram orientados quanto ao propósito da pesquisa e, caso aceitassem a participação, assinavam o Termo de Esclarecimento Livre e Esclarecido (TCLE), o qual dispunha de todas as informações com linguagem usual e de forma clara. Em seguida, eram coletados os dados referentes à pesquisa.

Os dados coletados foram então organizados no programa Statistical Package for the Social Sciences (SPSS), versão 22.0, para a construção de tabelas e análise descritiva.

Seguindo a Resolução $\mathrm{n}$-466, de 12 de dezembro de 2012, do Conselho Nacional de Saúde, que trata das normas para pesquisa envolvendo seres humanos, o presente estudo cumpre todos os seus aspectos éticos ${ }^{(7)}$. Esta pesquisa foi submetida à análise do comitê de 
ética em pesquisa do Hospital Universitário Onofre Lopes (HUOL), também localizado em Natal/RN e obteve parecer favorável, sob protocolo 437/2010.

\section{RESULTADOS E DISCUSSÃO}

De acordo com os dados obtidos no estudo, realizou-se a caracterização sociodemográfica com 125 vítimas de acidentes de trânsito, sendo $80,8 \%$ do sexo masculino, $63,2 \%$ com idade entre $18-35$ anos e escolaridade de $49,6 \%$ no ensino médio como demonstrado na Tabela 1.

Tabela 1 - Dados de caracterização das vítimas atendidas pelo SAMU 192 quanto ao sexo, faixa etária e grau de escolaridade. Natal, RN, Brasil, 2017.

\begin{tabular}{lll}
\hline Caracterização da vítimas & $\mathbf{n}$ & \% \\
Sexo & 101 & 80,8 \\
Masculino & 24 & 19,2 \\
Feminino & & 63,2 \\
Faixa etária & 79 & 32,8 \\
18 a 35 anos & 41 & 4,0 \\
36 a 67 anos & 5 & 4,0 \\
67 a 73 anos & & 39,8 \\
Grau de Escolaridade & 5 & 49,6 \\
Não Alfabetizado & 49 & 6,4 \\
Ensino Fundamental (I e II) & 62 & 0,8 \\
Ensino Médio & 8 & $\mathbf{1 0 0 , 0}$ \\
Ensino Superior & 1 & 125 \\
Não Informado & & \\
TOTAL & & \\
\hline Fonte: Dados da pesquisa, & & \\
\hline
\end{tabular}

Fonte: Dados da pesquisa, 2017.

Estudos realizados no Piauí( ${ }^{(2)}$ evidenciam a prevalência do sexo masculino, em torno de $71,8 \%$, com média de idade de 24 a 29 anos, em que os tipos de suporte encaminhados para os atendimentos das vítimas de acidentes de trânsito foram predominantemente a Unidade de Suporte Básico (USB) com 90\% das ocorrências e $10 \%$ atendidas pela Unidade de Suporte Avançado (USA), corroborando com os achados do presente estudo, onde dentre as ocorrências solicitadas, foram utilizadas para transporte e atendimento da vítima $87,2 \%$ as Unidade de Suporte Básico de Vida (USB), sendo apenas 7,2\% dos atendimentos realizados em Unidade de Suporte Avançado (USA). Com relação ao tempo de espera da vítima para ser socorrida, pode-se evidenciar que $42,4 \%$ dos pacientes foram atendidos entre cinco a 20 minutos e que apenas $1,6 \%$ tiveram tempo de espera superior a três horas.

Dados como estes confirmam as evidências mostradas nas literaturas e permitem acompanhar o crescimento na participação da mulher, ainda que não tão significativa, nos acidentes de trânsito, relacionado à evolução do processo de trabalho e suas conquistas, como maior porcentagem de mulheres procurando os serviços de autoescola, aprendendo a dirigir e aquisição do próprio carro, ainda que mais prudentes que os homens.

A Organização Mundial da Saúde (OMS), aponta que, em 2030, serão 2,4 milhões de pessoas mortas por AT. Os dados ainda mostram que, em países com rendimentos baixos ou médios, onde não há investimentos voltados para segurança nas vias públicas, os números podem ser ainda mais estarrecedores, a exemplo disso, o Brasil $^{(8)}$.

A predominância de vítimas acidentadas do sexo masculino atribui-se ao menosprezo de riscos durante a condução do veículo, evidenciando a personalidade masculina de se auto afirmar melhor que as mulheres Além disso, as mulheres são mais atenciosas e se arriscam menos, evitando ações imprudentes ${ }^{(9)}$.

Nem todas as vítimas terminam em morte, mas violência no trânsito representa um grau de extremidade amplo, se comparado a outros fatores de mortalidade da população. Desse modo, o conhecimento desta realidade é de fundamental 
importância para alertar os gestores públicos quanto à elaboração de políticas públicas voltadas à redução dos índices de acidentes fatais. Os profissionais dos serviços de saúde devem desempenhar um papel essencial frente a essa situação, sendo a educação em saúde, uma ferramenta importante para a efetivação do cuidado ${ }^{(3)}$.

Outro ponto relevante da pesquisa é a faixa etária das vítimas. Pode-se perceber que a maioria delas possuía idade entre 18 a 35 anos, o que se configura como preocupante, pois trata-se de população predominantemente jovem e com ensino. Pode-se perceber a existência de uma concentração maior de vítimas nessa faixa etária, sugerindo que os homens jovens são quem mais se arriscam no trânsito, devendo averiguar outros fatores condicionantes como o uso de álcool e $\operatorname{drogas}^{(10)}$.

Já relacionado à escolaridade, as prevalências um pouco maiores estiveram naqueles com ensino médio ou mais, o evidenciando um maior poder aquisitivo para o uso de veículos automotores privados ${ }^{(11)}$.

Há uma contradição quando se trata de escolaridade. Em regra, quanto maior o nível de conhecimento, deveria existir maior atenção ao que concernem às questões relacionadas ao trânsito. Porém, esse não parece ser um fator condicionante na minimização dos agravos. A escola é fundamental, não somente como instituição educadora, mas também ao favorecer adaptação dos jovens à disciplina e ao respeito às leis e normas vigentes para o bem comum ${ }^{(12)}$.

Tabela 2 - Caracterização do atendimento às vítimas atendidas pelo SAMU 192 RN quanto ao tipo de veículo de transporte utilizado e o tempo resposta. Natal, RN, Brasil, 2017.

\begin{tabular}{|c|c|c|}
\hline Caracterização do atendimento & $\mathbf{n}$ & $\%$ \\
\hline \multicolumn{3}{|l|}{ Veículo de transporte } \\
\hline USA & 9 & 7,2 \\
\hline USB & 109 & 87,2 \\
\hline Não informado & 7 & 5,6 \\
\hline \multicolumn{3}{|l|}{ Tempo resposta } \\
\hline 5 a $20 \min$ & 53 & 42,4 \\
\hline 21 a $45 \mathrm{~min}$ & 37 & 29,6 \\
\hline 46 a $360 \mathrm{~min}$ & 25 & 20,0 \\
\hline Acima de $360 \mathrm{~min}$ & 2 & 1,6 \\
\hline Não Informado & 8 & 6,4 \\
\hline Total & 125 & 100,0 \\
\hline
\end{tabular}

Fonte: Dados da pesquisa, 2017.

Com relação ao tipo de acidente sofrido pelas vítimas entrevistadas, a Tabela 2 mostra que a maioria desses foram ocasionados por colisões (carro $x$ carro, carro $x$ caminhão, carro $x$ trem, carro $x$ moto, moto $x$ ônibus, moto $x$ caminhão) perfazendo um total de $84 \%$, seguidos por $1,6 \%$ vítimas de capotamento, $2,4 \%$ quedas de motocicletas e $12 \%$ dos entrevistados foram vítimas de atropelamento.

O transporte mais utilizado foi a USB. Segundo a portaria GM/MS № 356, de 8 de abril de 2013, o registro total de todas as ocorrências atendidas pela equipe de suporte básico de vida deverá representar $80 \%$ do total das chamadas recebidas pela central de regulação das urgências com envio de unidade móvel e 30\% deverá ser atendido pelo suporte avançado de vida ${ }^{(13)}$. Assim sendo, o estudo em questão segue a tendência do que preconiza o Ministério da Saúde.
Com relação ao atendimento do SAMU 192 $\mathrm{RN}$, pode-se evidenciar que a maioria das ocorrências foi atendida em tempo hábil, cerca de 5 a 20 minutos de espera do paciente. Existem três picos de morte: no primeiro pico, observa-se a mortalidade em segundos ou minutos pós trauma; o segundo, costuma estar presente na primeira ou segunda hora pós trauma e é denominado por Hora de Ouro; já no terceiro pico, a morte ocorre em dias ou semana pós trauma ${ }^{(14)}$. Quanto mais rápido o atendimento for realizado, maior a chance de sobrevida da vítima.

O tempo transcorrido para o início dos primeiros socorros, a estabilização no local da cena e a chegada do paciente ao hospital são fatores decisivos, oportunizando uma maior possibilidade de sobrevida. Assim, percebemos a importância da existência da equipe de Atendimento Pré-Hospitalar (APH) bem 
qualificada, preparada para o cuidado de urgência às vítimas de trauma nas vias e logradouros públicos. O profissional deve ser capaz de realizar uma minuciosa avaliação da gravidade do trauma, empenhando-se em reduzir ao máximo as complicações ${ }^{(14)}$.

Tabela 3 - Caracterização do atendimento das vítimas assistidas pelo SAMU 192 RN quanto ao tipo de acidente de trânsito. Natal, RN, Brasil, 2017.

\begin{tabular}{lcc}
\hline Caracterização do tipo de acidente & $\mathbf{n}$ & $\%$ \\
Colisão & 105 & 84 \\
Atropelamento & 15 & 12 \\
Queda de motocicleta & 3 & 2,4 \\
Capotamento & 2 & 1,6 \\
Total & $\mathbf{1 2 5}$ & $\mathbf{1 0 0}$ \\
\hline
\end{tabular}

Fonte: Dados da pesquisa, 2017.

Como apresentado, a maioria dos AT foram ocasionados por colisões, porém, um dado preocupante é o percentual de atropelamento. Apesar das colisões serem mais frequentes, observamos que os acidentes envolvendo pedestres tendem a ser mais graves. Estudos realizados apresentam que os atropelamentos, apesar de serem em menor quantidade, são os responsáveis pela maior quantidade de óbitos decorrentes de trauma ${ }^{(15)}$. Os pedestres, por sofrerem o impacto direto em caso de atropelamentos, são as vítimas com maior probabilidade de serem internadas e morrerem após terem sofrido um acidente, configurando-se no grupo mais vulnerável em relação aos motoristas e passageiros ${ }^{(12)}$.

Esta classificação se torna essencial para que possamos analisar cada vítima de acordo com sua qualidade de usuário da via pública, identificando-o como condutor ou passageiro de automóvel, motociclista ou pedestre atropelado por algum destes veículos ${ }^{(14)}$.

Tabela 4 - Caracterização do atendimento das vítimas atendidas pelo SAMU 192 RN quanto ao dia da semana que ocorreu o acidente de trânsito. Natal, RN, Brasil, 2017.

\begin{tabular}{lcc}
\hline Caracterização Temporal & $\mathbf{n}$ & $\%$ \\
Dias da semana & & 16,6 \\
Domingo & 25 & 16,6 \\
Segunda-feira & 20 & 2,0 \\
Terça-feira & 20 & 8,0 \\
Quarta-feira & 19 & 10,4 \\
Quinta-feira & 15 & 20,0 \\
Sexta-feira & 13 & 15,2 \\
Sábado & 10 & 2,4 \\
Ignorado & 3 & 100,0 \\
Total & 125 & 2 \\
\hline
\end{tabular}

Fonte: Dados da pesquisa, 2017.

Os AT foram caracterizados também com relação ao turno e dia da semana em que ocorreram. Pode-se identificar que 25 (20,0\%) ocorreram principalmente no início do final de semana, ou seja, sextas e sábados, e que a grande maioria deles ocorreu no turno matutino. A Tabela 4 aponta os dados referentes aos dias da semana em que os atendimentos foram realizados.

Analisou-se ainda que a maioria dos acidentes ocorriam nos finais de semana, em especial nas sextas e domingos. O turno da noite, apesar de não se apresentar como maioria, chama atenção. Em Londrina, Paraná, no período de janeiro a junho de 1996, identificou-se que o horário de maior média de vítimas/dia variava conforme os dias da semana. O período com o maior número de vítimas foi das 20 às 21 horas $^{(15)}$.

O acúmulo de óbitos no período da noite sugere que essa ocorrência se deva ao cansaço, que é maior no final do dia aliado a esses fatos. 
Podemos citar também que, no período noturno e da madrugada, ocorrem o desrespeito aos semáforos, excesso de velocidade, dificuldade de atendimento imediato às vítimas de acidentes de trânsito, entre outros fatores ${ }^{(12)}$.

Sugere-se que os dados apresentados com altos valores para a variável "manhã" e "tarde" ocorreram, pois, a coleta era realizada, em sua maioria, no turno matutino e vespertino em que a maioria dos pacientes, vítimas de AT no período da noite, já haviam sido atendidas, estabilizadas e encaminhadas para outros setores do hospital, caracterizando, por sua vez, um falso positivo às variáveis. Ademais, ainda contamos com a particularidade de que a maioria dos entrevistados não informou o turno do ocorrido.

Muitos fatores podem estar relacionados aos dados encontrados, o uso de álcool, o sono prejudicado, o excesso de velocidade, uma vez que o tráfego de veículos costuma diminuir nos finais de semana. Os resultados nos confirmam a importância da utilização desses índices de avaliação como ferramentas indispensáveis na prestação de uma assistência individualizada, voltada para as necessidades dos pacientes.

Em nosso país e, em especial no Nordeste, há uma escassez de estudos que retratem a gravidade do trauma e das lesões decorrentes desses eventos. Muito se fala sobre eles, mas pouco se conhece acerca da forma correta de se realizar a triagem e avaliação dos pacientes e, assim, minimizar as consequências advindas do trauma $^{(14)}$.

O trauma é uma das mais importantes causas de morbimortalidade e incapacidade em longo prazo na população adulta jovem mundial. Constitui um dos principais problemas de saúde pública em muitos países, independente do desenvolvimento socioeconômico. No Brasil, poucos são os centros públicos de referência no atendimento a esse tipo de paciente ${ }^{(16-19)}$.

Figura 1 - Caracterização das vítimas atendidas pelo SAMU 192 RN, quanto à parte do corpo afetada. Natal, RN, Brasil, 2017.

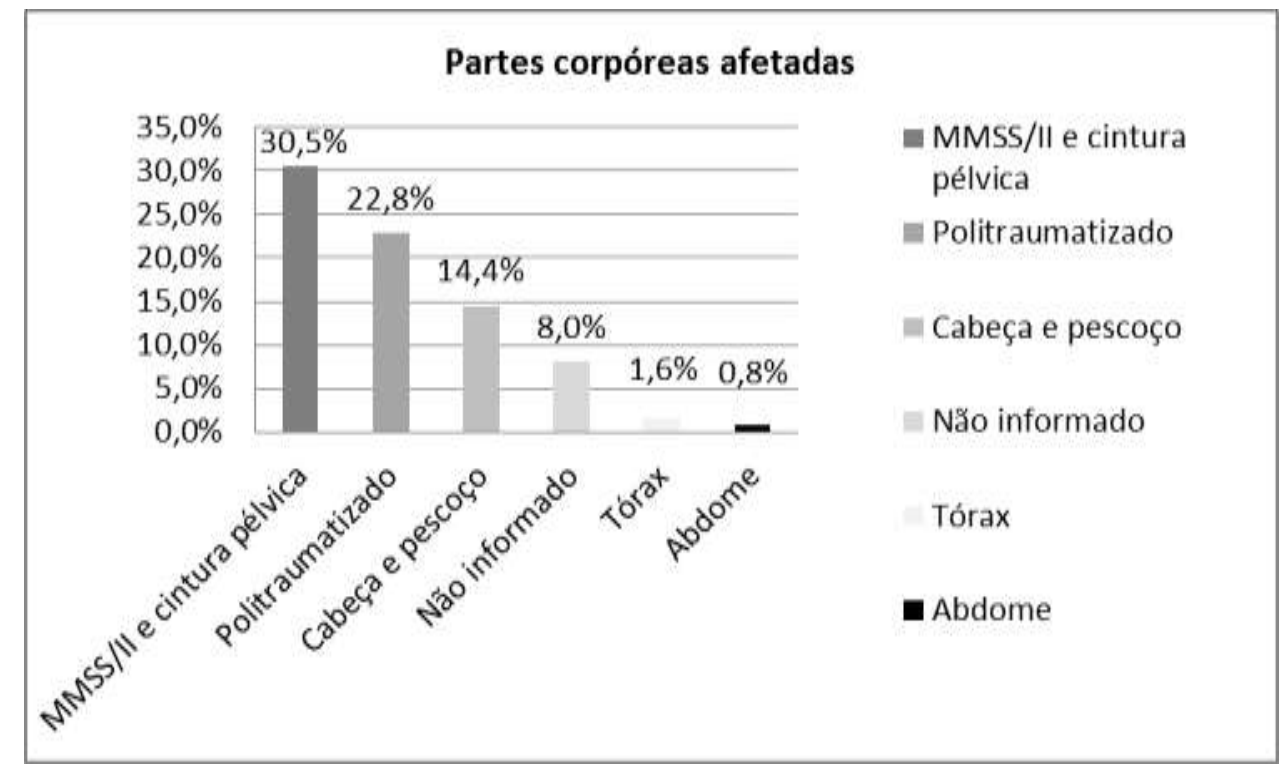

Fonte: Dados da pesquisa, 2017.

Por fim, a partir dos dados obtidos, foi possível avaliar a gravidade do acidente a partir da extensão das lesões sofridas pelas vítimas, verificando-se que $61(48,8 \%)$ dos entrevistados tiveram seus membros superiores e/ou inferiores (MMSS/II) afetados e/ou cintura pélvica, seguidos por $38(30,4 \%)$ que sofreram politrauma, ou seja, várias regiões foram afetadas.

Ao analisarmos os dados, grande parte dos usuários foram afetados nas regiões dos MMSS/II e/ou cintura pélvica e a segunda maior parte trata-se de pacientes politraumatizados.
Independentemente da origem do trauma, uma pessoa pode apresentar lesões graves, necessitando de intervenção apropriada para evitar a morte e sequelas advindas desse evento. Desta feita, o cuidado às vítimas deve ser iniciado no atendimento pré-hospitalar. O manejo inicial desses pacientes apresenta algumas peculiaridades que requerem de toda a equipe de saúde, atuação específica ${ }^{(14)}$.

A realidade vista neste estudo ainda se configura a partir de dados significativos, o que reforça a necessidade de uma atenção maior dos 
gestores públicos com vistas à divulgação de medidas preventivas capazes de minimizar o número alarmante de ocorrências. A educação no trânsito, estimulada principalmente pelos profissionais de saúde, torna-se fundamental para mudar esse prognóstico.

\section{CONCLUSÃO}

Nota-se um predomínio de pacientes homens com faixa etária de 28 a 35 anos de idade e média de escolaridade em ensino médio. As unidades mais utilizadas para atendimento são as Unidades de Suporte Básico (USB) e o tempo resposta de 5 a 20 minutos, onde a caracterização do tipo do acidente mostrou-se mais atuante em colisões, representando $84 \%$, seguido de atropelamento, queda de motocicleta e capotamento. A caracterização temporal explicita aos finais de semana e as áreas como membros inferiores e cintura pélvica como a mais atingida.

Os achados permitiram caracterizar o perfil epidemiológico das vítimas de acidentes de trânsito socorridas pelo Serviço de Atendimento Móvel de Urgência (SAMU), permitindo maiores reflexões sobre as prevenções dos acidentes, conscientização da população jovem caracterizada no estudo, bem como conhecimento da equipe de atendimento ao paciente vítima de AT.

Estudos como este refutam a importância de políticas de prevenções mais atuantes que reflitam sobre a redução dos índices de acidentes e, consequentemente, de gastos públicos com reabilitação. É imprescindível conhecer a realidade na qual o paciente está inserido e identificar fragilidades e potencialidades para que o processo de educação em saúde seja efetuado de forma significativa e compreensível na comunicação.

Ainda assim, demonstra a necessidade de mais estudos na área onde possam trazer novos questionamentos para que se tenha como fim, um menor tempo de atendimento, redução do número de óbitos e necessidade constante de atualização de informações e manejo com o paciente, realizados pela equipe.

\section{REFERÊNCIAS}

1. Organização Mundial da Saúde (OMS). Causas externas de morbidade e de mortalidade. In: Classificação estatística internacional de doenças e problemas relacionados à saúde 10 a edição. São Paulo; OMS; 2007. p.969-1011.

2. Cavalcante AKCB, Holanda VM, Rocha CFM, Cavalcante SW, Sousa JPR, Sousa FHR. Perfil dos acidentes de trânsito atendidos por serviço préhospitalar móvel. Rev Baiana Enferm [Internet]. 2015 [citado em 2018 mar 7];29(2):135-45. Disponível em: https://portalseer.ufba.br/index.php/enfermage m/article/viewFile/12656/pdf 125

3. Luchtemberg MN, Pires DEP. Enfermeiros do Serviço de Atendimento Móvel de Urgência: perfil e atividades desenvolvidas. Rev Bras Enferm [Internet]. 2016 [citado em 2018 mar 7];69(2):194-201. Disponível em: http://www.scielo.br/pdf/reben/v69n2/00347167-reben-69-02-0213.pdf

4. Ministério da Saúde (Brasil). Política Nacional de Atenção às Urgências. Brasília, DF; 2011. Disponível em: http://bvsms.saude.gov.br/bvs/saudelegis/gm/20 11/prt2395_11_10_2011.html.

5. Ministério da Saúde (Brasil), Portal da Saúde. O que é o SAMU 192? [Internet]. [citado em 2017 ago 14]. Disponível em: http://portalsaude.saude.gov.br/index.php/oministerio/principal/secretarias/951-sasraiz/dahu-raiz/forca-nacional-do-sus/l2-forcanacional-do-sus/13407-servico-de-atendimentomovel-de-urgencia-samu-192

6. Santana EMC, Nunes MN, Nascimento LFC. Acidentes de trânsito com motociclistas, no Estado de São Paulo (2005-2009): uma abordagem espacial. Hygeia [Internet]. 2013 [citado em 2017 ago 20];9(17):19-28. Disponível em:

http://www.seer.ufu.br/index.php/hygeia/article/ view/23100/13619

7. Ministério da Saúde (Brasil), Conselho Nacional de Saúde. Diretrizes e normas regulamentadoras de pesquisa envolvendo seres humanos. Resolução no 466, de 12 de dezembro de 2012 [Internet]. Brasília, DF; 2012. Disponível em: http://bvsms.saude.gov.br/bvs/saudelegis/cns/20 13/res0466 $12 \quad 12 \quad 2012 . h t m l$

8. Waiselfisz JJ. Mapa da violência 2013: acidentes de trânsito e motocicletas. Rio de Janeiro: CEBELA [Internet]; 2013 [citado em 2017 ago 14]. Disponível em: http://www.mapadaviolencia.org.br/pdf2013/ma pa2013 transito.pdf

9. Melo LMF, Lima ATA, Rodrigues ACS, Rodrigues LG, Ferreira LAR, Randow RM, et al. Perfil epidemiológico dos motociclistas acidentados no município de Manhuaçu - Minas Gerais. Anais do Io Seminário Científico da FACIG; 2015 out 29-31; Manhuaçu, MG [internet]. Manhuaçu; 2015. [citado em 2017 ago 20]. Disponível em: 
http://www.pensaracademico.facig.edu.br/index.ph p/semiariocientifico/article/viewFile\%20/274/241

10. Souza VDR, Cavenaghi S, Alves JED. Mapeamento dos óbitos por local de ocorrência dos acidentes de trânsito na cidade do Rio de Janeiro. Anais do 150 Encontro Nacional dos Estudos Populacionais; 2006 set 1-22; Caxumbu, MG [internet]. Caxambu; 2006. [citado em 2017 ago 20]. Disponível em: http://www.abep.org.br/publicacoes/index.php/a nais/article/view/1562/1525

11. Damacena GN, Malta DC, Boccolini CS, Souza Junior PRBD, Almeida WDSD, Ribeiro LS, et al. Consumo abusivo de álcool e envolvimento em acidentes de trânsito na população brasileira, 2013. Ciênc Saúde Coletiva [Internet]. 2016 [citado em 2017 ago 20];21(12):3777-86. Disponível em: http://www.scielo.br/pdf/csc/v21n12/1413-8123csc-21-12-3777.pdf

12. Abreu ANM, Jomar RT, Thomaz RGG, Guimarães RM, Lima JMB, Fiqueiró RFS. Impacto da Lei Seca na mortalidade por acidentes de trânsito. Rev Enferm [Internet]. 2012 [citado em 2017 ago 19];20(1):21-6. Disponível em: http://www.e-

publicacoes.ueri.br/index.php/enfermagemueri/a rticle/view/3970/2753

13. Ministério da Saúde (Brasil), Secretaria de atenção à saúde. Portaria no 356, de 8 de abril de 2013 [Internet]. Brasília, DF; 2013. Disponível em: http://bvsms.saude.gov.br/bvs/saudelegis/sas/20 13/prt0356 $0804 \quad 2013 . \mathrm{html}$

14. Santos SMJ, Souza MA, Rocha FL, Souza VP, Muniz MAS, Rodrigues JA. Caracterização dos fatores de risco para acidentes de trânsito em vítimas atendidas pelo serviço móvel de urgência. Rev Enferm UFPE [Internet]. 2016 [citado em 2018 mar 7];10(10):3819-24. Disponível em: file:///C:/Users/home/Downloads/11448-26357-

\section{1-PB.pdf}

15. Padovani C, Silva JM, Tanaka C. Perfil dos pacientes politraumatizados graves atendidos em um serviço público de referência. Arq Cienc Saude [Internet]. 2014 [citado em 2017 ago 20];21(3)415. Disponível em: http://repositorioracs.famerp.br/racs ol/vol-21-3/IDZ-610-(213)\%20jul-Set-2014.pdf

16. Dantas RAN, Costa IKF, Nóbrega WG, Dantas DV, Costa IKF, Torres GV. Ocorrências realizadas pelo serviço de atendimento móvel de urgência metropolitano. Rev Enferm UFPE [Internet]. 2014 [citado em 2016 set 6];8(4). Disponível em: https://periodicos.ufpe.br/revistas/revistaenferm agem/article/viewFile/9751/9867

17. Dias JMC, Lima MSM, Dantas RAN, Costa IKF, Leite JEL, Dantas DV. Perfil de atendimento do serviço pré-hospitalar móvel de urgência estadual. Cogitare Enferm. 2016;21(1). http://dx.doi.org/10.5380/ce.v21i1.42470

18. Sarmento SDG, Dantas RAN, Dantas DV, Oliveira SP, Henriques LMN, Costa IB. Profile of individuals with neurological disorders assisted by a prehospital mobile emergency care service. Cogitare Enferm [Internet]. 2017 [citado em 2017 jun 28];22(2). Disponível em: http://www.saude.ufpr.br/portal/revistacogitare/ wp-content/uploads/sites/28/2017/04/49698204169-1-PB.pdf

19. Dantas RAN, Torres GV, Salvetti MG, Dantas $D V$, Mendonça AEO. Instrument for assessing the quality of mobile emergency pre-hospital care: content validation. Rev Esc Enferm USP [Internet]. 2015 [citado em 2016 set 4];49(3). Disponível em: http://www.scielo.br/pdf/reeusp/v49n3/00806234-reeusp-49-03-0381.pdf

Nota: Este artigo possui financiamento pelo Edital Universal (2016) do Conselho Nacional de Desenvolvimento Científico e Tecnológico $(\mathrm{CNPq})$, regido pelo processo número: 403613/2016-7.

Recebido em: 10/10/2017

Aprovado em: 19/03/2018

Endereço de correspondência:

Rodrigo Assis Neves Dantas

Rua Petra Kelly, 61.

CEP: 59152-330 - Parnamirim/RN - Brasil

E-mail: rodrigoenf@yahoo.com.br 